\title{
INVESTIGATION OF MAGNITUDE EFFECT IN THE AGK3R CATALOGUE
}

\author{
J. L. SCHOMBERT and T. E. CORBIN \\ U.S. Naval Observatory, Washington, D.C., U.S.A.
}

\begin{abstract}
The AGK3R Catalogue and the FK4 observations made in connection with this catalogue were examined for magnitude effects. Each observatory's observations were compared to the mean position for all stars. A comparison was also made with the stars grouped by spectral type. The magnitude effects found were very small.
\end{abstract}

\section{Introduction}

The AGK3R Catalogue is a catalogue of 21499 stars between $+90^{\circ}$ and $-5^{\circ}$ declination. These stars were observed between 1956 and 1964 with eleven transit instruments at ten different observatories, two instruments being located at the U.S. Naval Observatory. Usually, a star was observed twice with five instruments, although there were many cases where four observations were made at one observatory. The observations were reported to the U.S. Naval Observatory where they were analyzed and each participant's observations reduced to the fundamental system. A final catalogue was then formed. It is these observatory means, for both the AGK3R stars and the FK4 stars, that have been examined for the existence of residual magnitude effects.

\section{Comparison of Observatory Means}

After each observatory's observations had been reduced to the FK4 system, an average for each star was formed by use of an appropriate weighting factor, and a final catalogue of positions was determined. For each star observed by each instrument, the observed position was reduced to the epoch of the final catalogue using proper motions from the Smithsonian Astrophysical Observatory Catalog. Differences, (Obs. - Cat.), were then formed for all stars, AGK3R and FK4. These differences were solved by a least squares method for a constant displacement and a rate with magnitude using the following equations:

where

$$
\text { Constant displacement }=\overline{\Delta \alpha}=\frac{\sum \Delta \alpha}{N},
$$

$\Delta \alpha=$ difference between observatory mean and final position, (Obs. - Cat.)

$N=$ number of stars

and

$$
\text { Rate } / \text { magnitude }=\Delta \alpha_{m}=\frac{\sum(\Delta \alpha \Delta m)}{\sum(\Delta m)^{2}}
$$


where

$$
\begin{aligned}
\Delta m= & \text { difference between a star's magnitude and the average } \\
& \text { magnitude for all stars of that observatory. }
\end{aligned}
$$

Similar equations were used for declination. The results of this solution are shown in the graphs of Figures 1 through 6 and in Tables I and II.

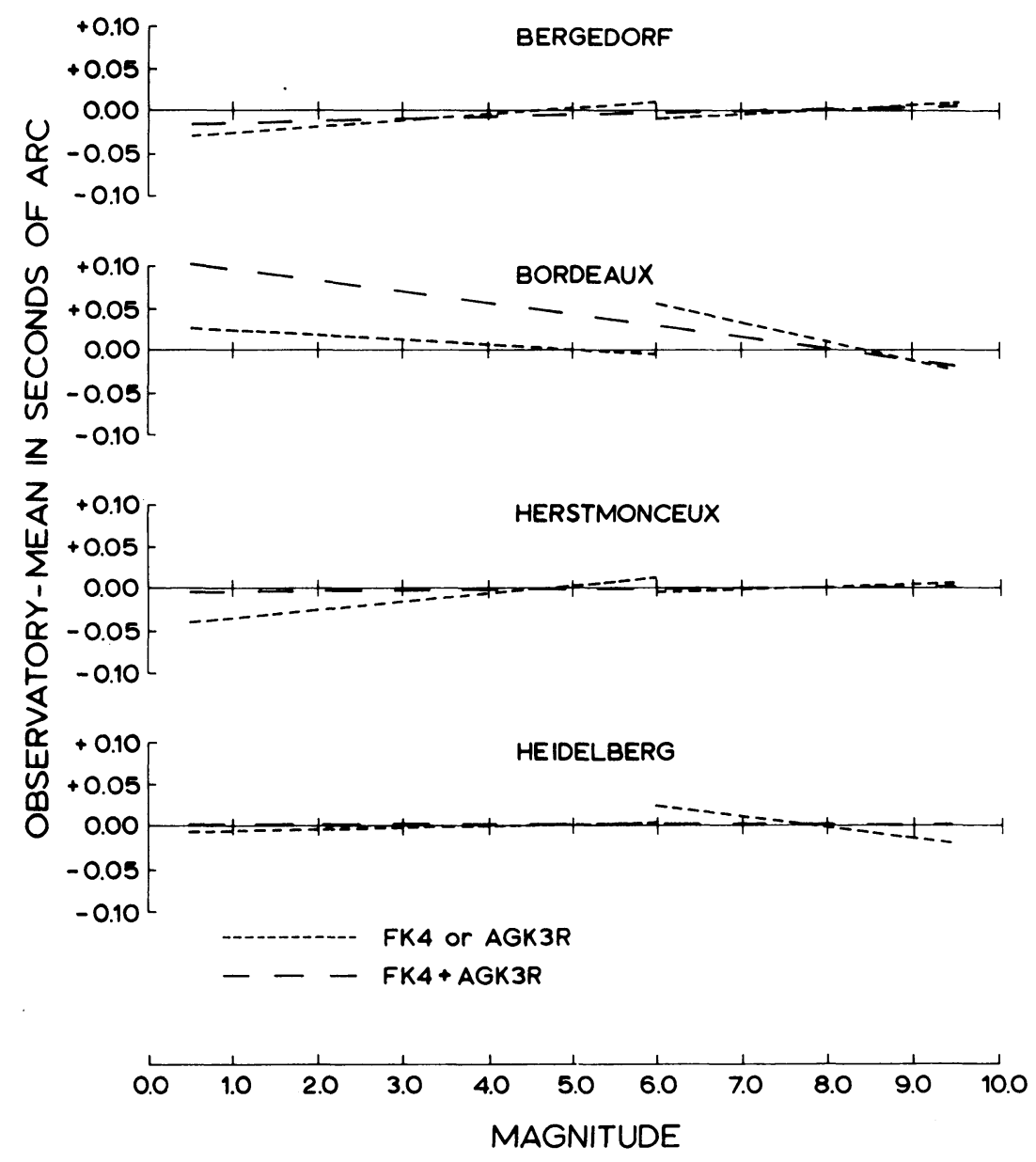

Fig. 1. Right ascension.

For the most part the slopes are quite small, however, for a few observatories there seems to be a small magnitude effect. The smaller ones are about the same size as their mean errors, whereas the larger ones are about 2 to 4 times the mean error. In the worst case the rate is about 0 ". 1 over an 8 mag. range. 


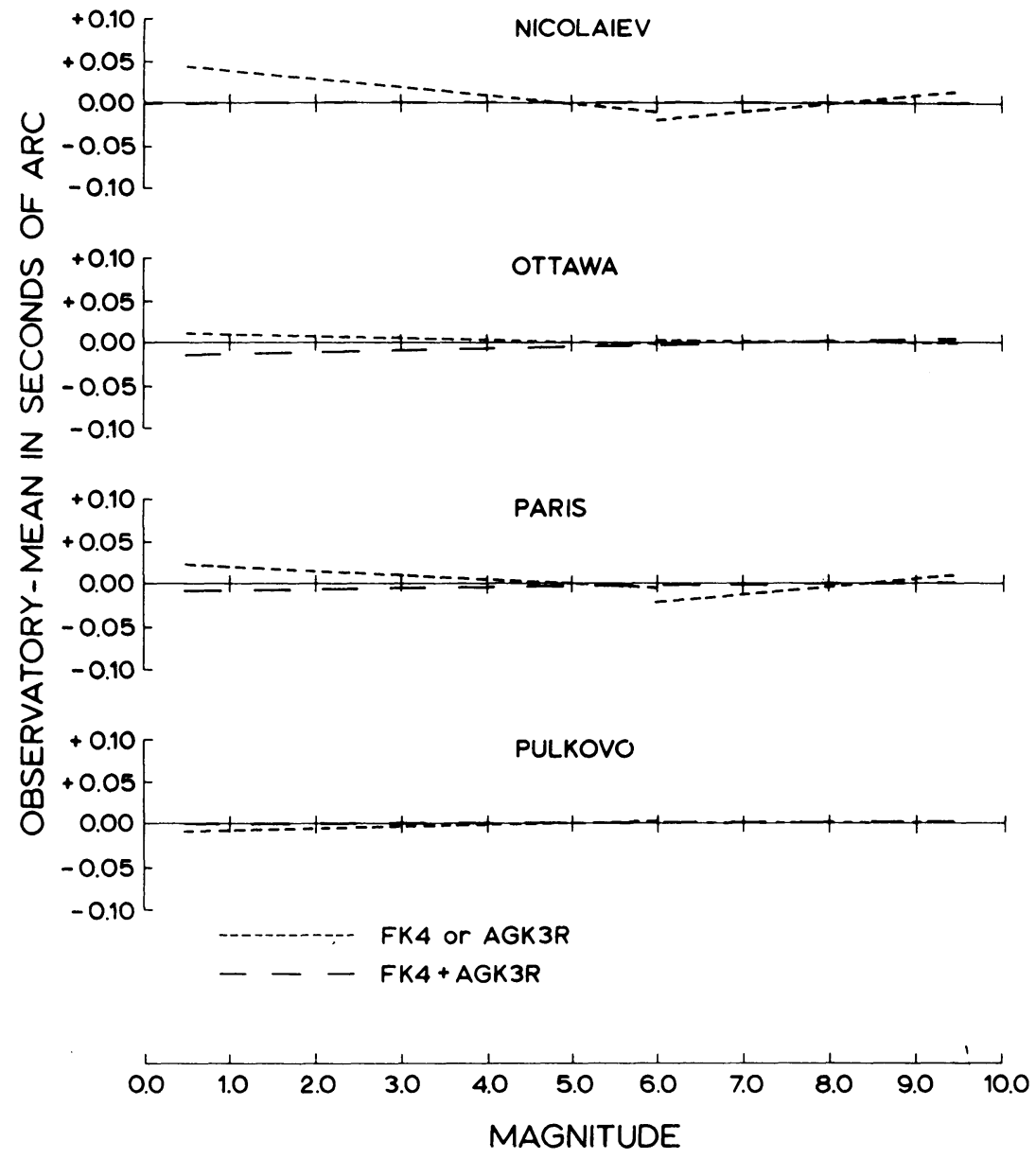

Fig. 2. Right ascension. 

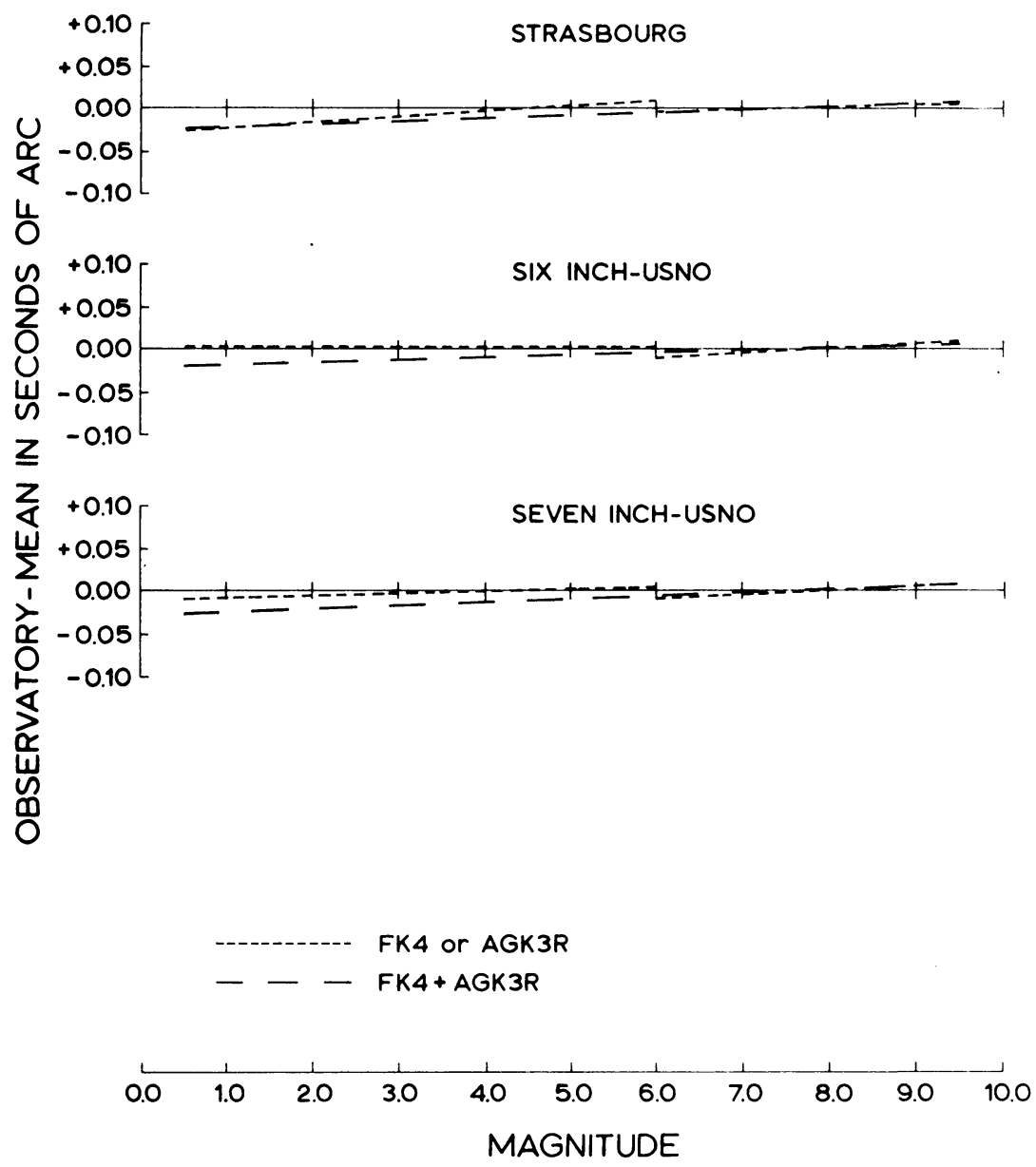

Fig. 3. Right ascension. 


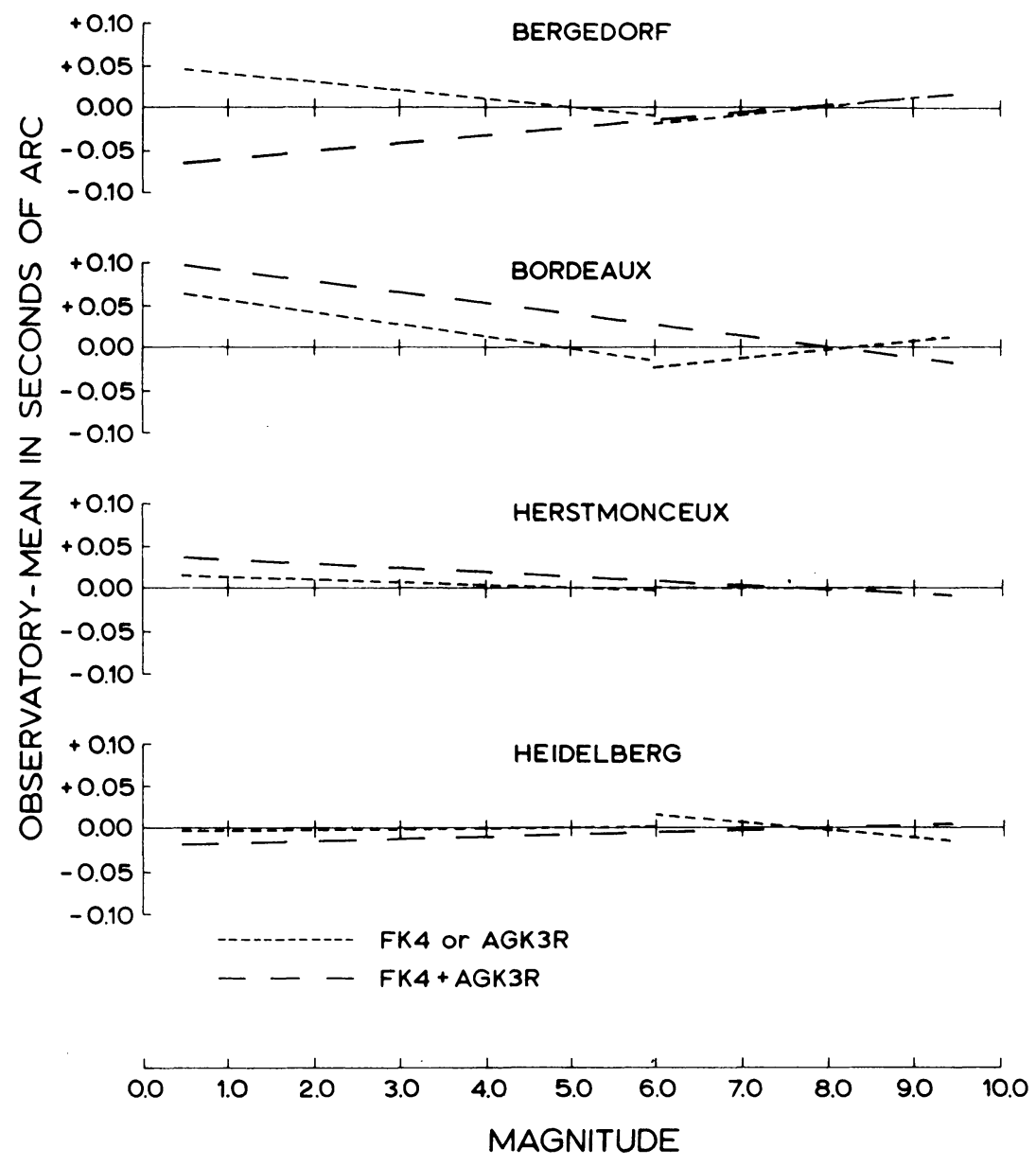

Fig. 4. Declination. 


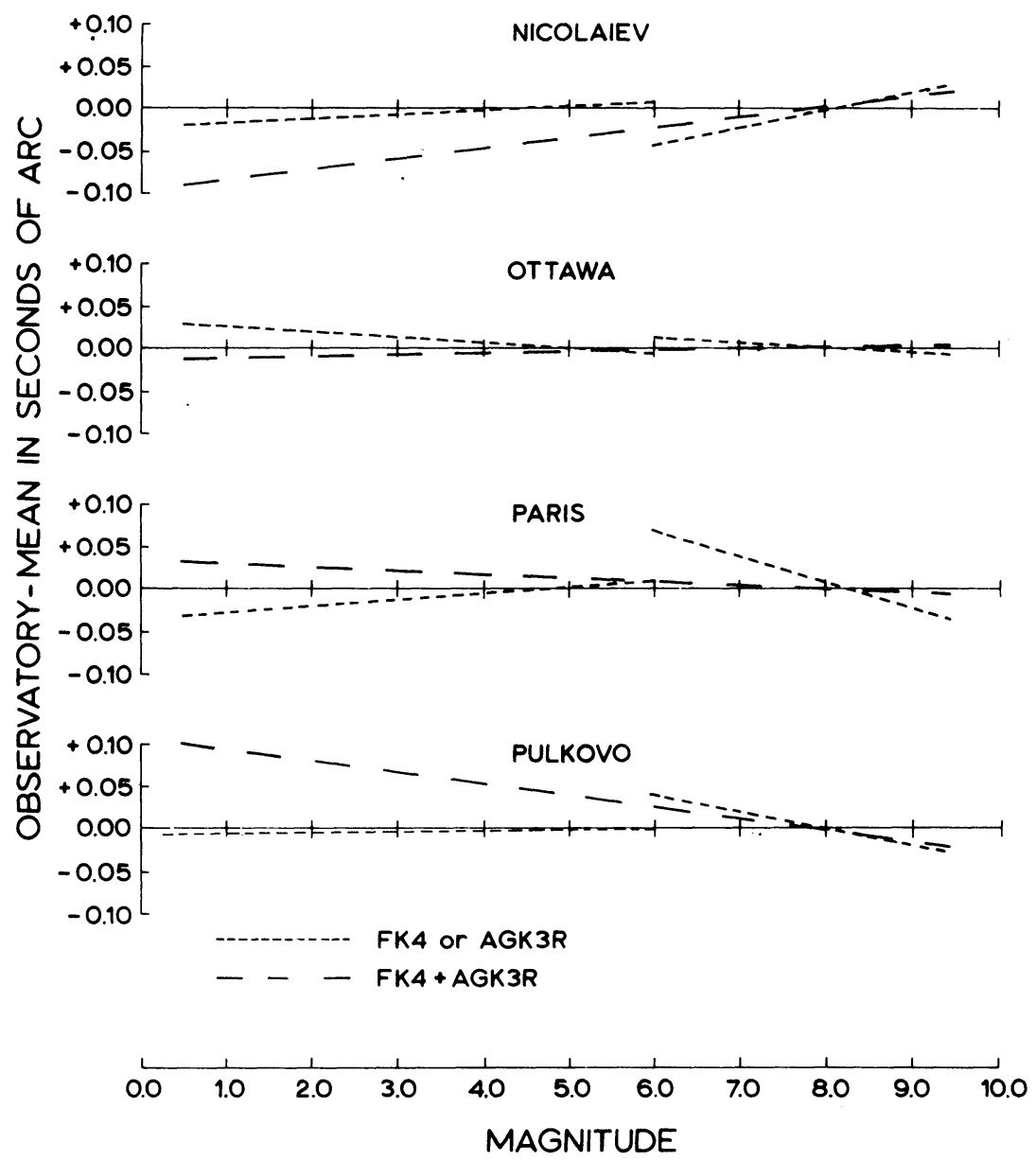

Fig. 5. Declination. 

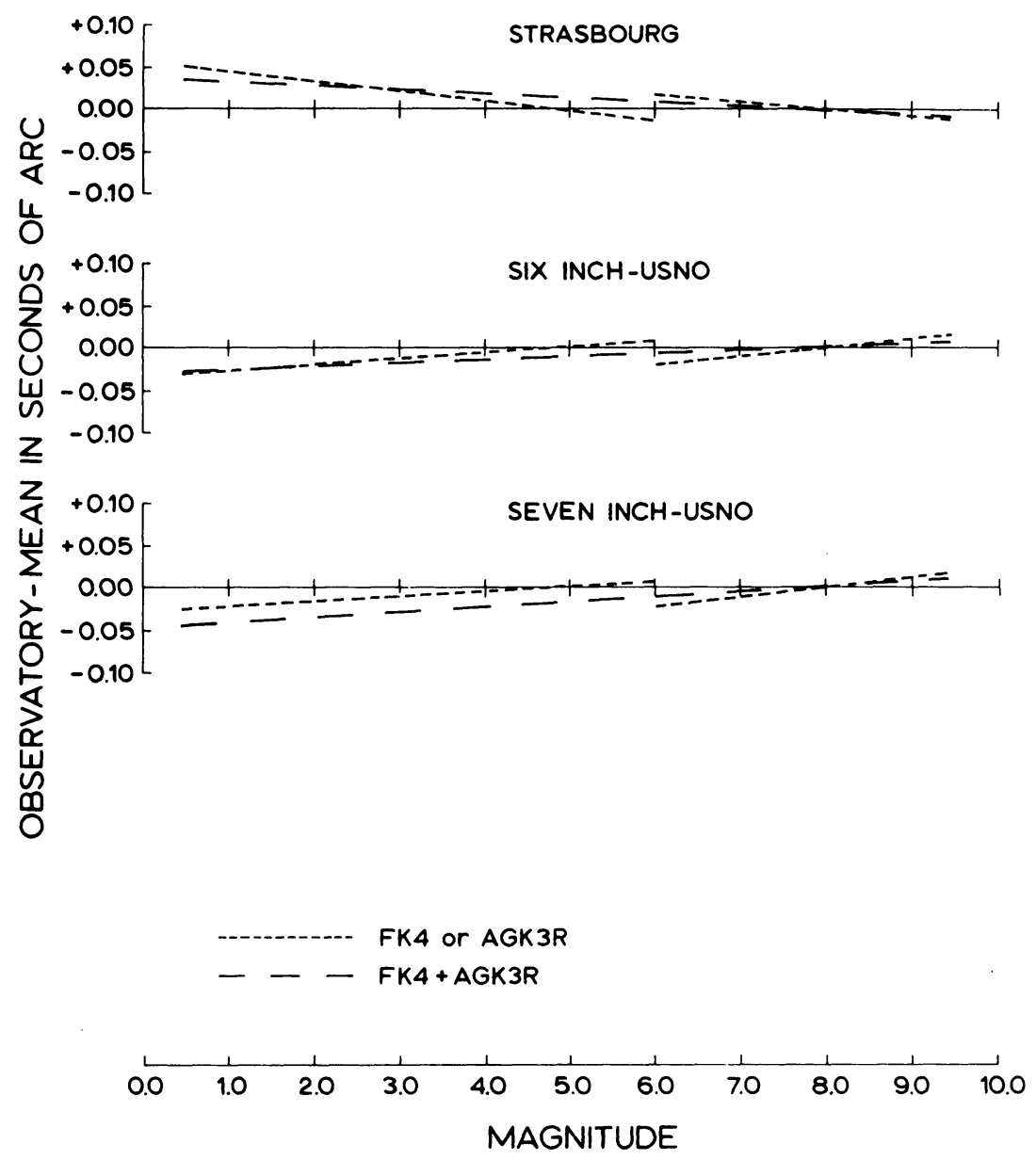

Fig. 6. Declination. 
TABLE I

Observatory - Catalogue

\begin{tabular}{|c|c|c|c|c|c|c|c|}
\hline \multirow[b]{2}{*}{ Observatory } & \multicolumn{2}{|c|}{$\mathrm{AGK} 3 \mathrm{R}+\mathrm{FK} 4$} & & \multicolumn{2}{|l|}{ AGK3R } & \multicolumn{2}{|l|}{ FK4 } \\
\hline & $\overline{\Delta \alpha}$ & $\Delta \alpha_{m}$ & & $\overline{\Delta \alpha}$ & $\Delta \alpha_{m}$ & $\overline{\Delta \alpha}$ & $\Delta \alpha_{m}$ \\
\hline Bergedorf & -0.0009 & +0.0027 & & -0.0011 & +0.0057 & +0.0002 & +0.0070 \\
\hline Bordeaux & -0.0335 & -0.0138 & & -0.0374 & -0.0225 & +0.0044 & -0.0060 \\
\hline Herstmonceux & -0.0056 & +0.0015 & & -0.0057 & +0.0033 & -0.0015 & +0.0108 \\
\hline Heidelberg & +0.0105 & -0.0004 & & +0.0120 & -0.0126 & -0.0039 & -0.0006 \\
\hline Nicolaiev & -0.0074 & +0.0018 & . & -0.0080 & +0.0102 & +0.0045 & -0.0057 \\
\hline Ottawa & +0.0111 & +0.0022 & & +0.0134 & -0.0009 & +0.0018 & -0.0036 \\
\hline Paris & -0.0006 & +0.0012 & & -0.0009 & +0.0096 & +0.0023 & -0.0051 \\
\hline Pulkovo & +0.0015 & +0.0004 & & +0.0017 & +0.0003 & +0.0008 & +0.0022 \\
\hline Strasbourg & +0.0015 & +0.0034 & & +0.0024 & +0.0028 & -0.0087 & +0.0063 \\
\hline USNO 6-in. & +0.0068 & +0.0027 & & +0.0074 & +0.0057 & +0.0015 & 0.0000 \\
\hline USNO 7 -in. & +0.0113 & +0.0039 & & +0.0120 & +0.0050 & +0.0009 & +0.0024 \\
\hline
\end{tabular}

TABLE II

Observatory - Catalogue

\begin{tabular}{|c|c|c|c|c|c|c|}
\hline \multirow[b]{2}{*}{ Observatory } & \multicolumn{2}{|c|}{$\mathrm{AGK} 3 \mathrm{R}+\mathrm{FK} 4$} & \multicolumn{2}{|l|}{ AGK3R } & \multicolumn{2}{|l|}{ FK4 } \\
\hline & $\overline{\Delta \delta}$ & $\Delta \delta_{m}$ & $\overline{\Delta \delta}$ & $\Delta \delta_{m}$ & $\overline{\Delta \delta}$ & $\Delta \delta_{m}$ \\
\hline Bergedorf & +0.0293 & +0.0089 & +0.0316 & +0.0104 & -0.0018 & -0.0102 \\
\hline Bordeaux & -0.0666 & -0.0124 & -0.0735 & +0.0103 & +0.0006 & -0.0151 \\
\hline Herstmonceux & -0.0282 & -0.0042 & -0.0296 & +0.0006 & -0.0064 & -0.0039 \\
\hline Heidelberg & +0.0232 & +0.0022 & +0.0252 & -0.0086 & +0.0031 & +0.0018 \\
\hline Nicolaiev & +0.0251 & +0.0128 & +0.0264 & +0.0208 & +0.0013 & +0.0054 \\
\hline Ottawa & +0.0132 & +0.0023 & +0.0163 & -0.0056 & +0.0006 & -0.0070 \\
\hline Paris & +0.0153 & -0.0041 & +0.0166 & -0.0296 & +0.0039 & +0.0073 \\
\hline Pulkovo & -0.0305 & -0.0121 & -0.0315 & -0.0193 & -0.0103 & +0.0016 \\
\hline Strasbourg & +0.0058 & -0.0049 & +0.0052 & -0.0083 & +0.0117 & -0.0118 \\
\hline USNO 6-in. & +0.0031 & +0.0038 & +0.0033 & +0.0100 & +0.0014 & +0.0060 \\
\hline USNO 7-in. & +0.0090 & +0.0060 & +0.0097 & +0.0114 & +0.0004 & +0.0057 \\
\hline
\end{tabular}

\section{Comparison by Spectral Type}

The differences were also grouped by spectral type. $\mathrm{K}, \mathrm{M}$, and $\mathrm{R}$ stars formed the red group and $\mathrm{O}, \mathrm{B}, \mathrm{A}, \mathrm{F}$ and $\mathrm{G}$ the blue. The same type solution was made for these groups and the results are given in Tables III and IV. As can be seen, there seems to be no significant relationship with color.

\section{Comparison with the FK4}

A comparison was made between the FK4 itself and the observational means of the 


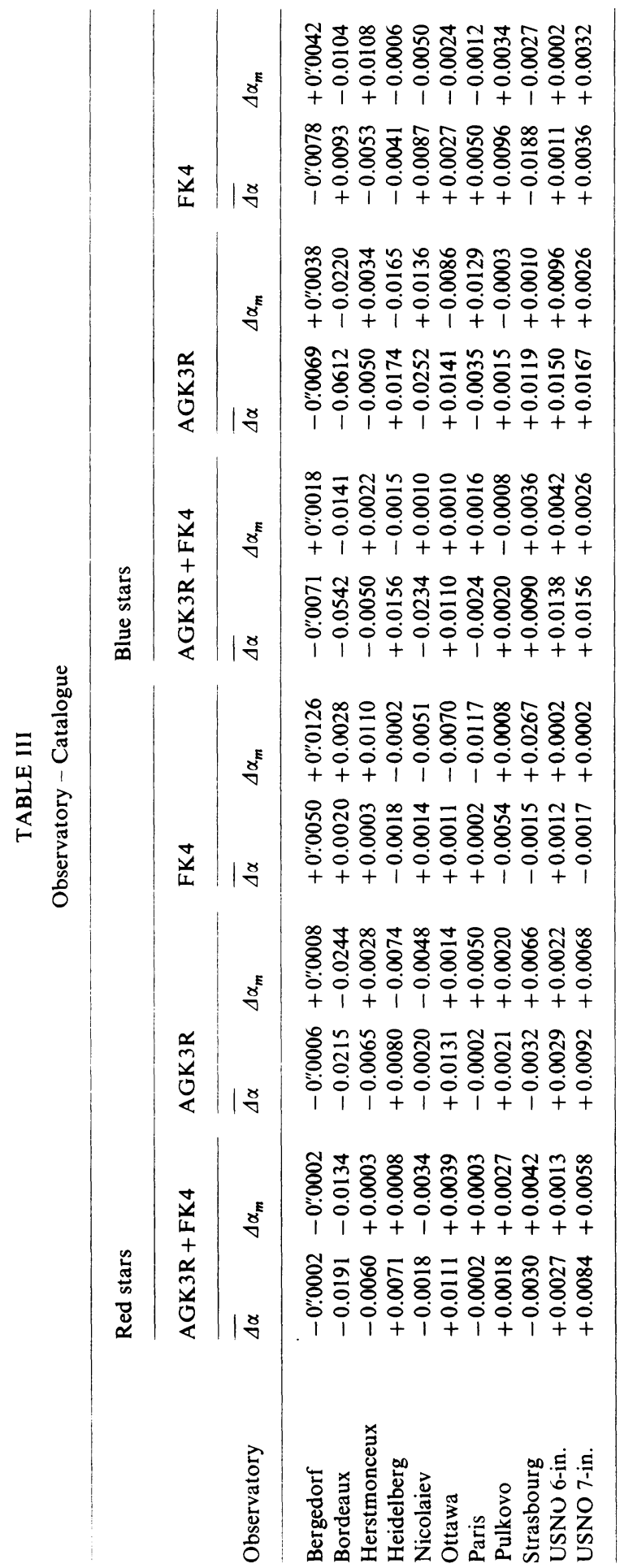




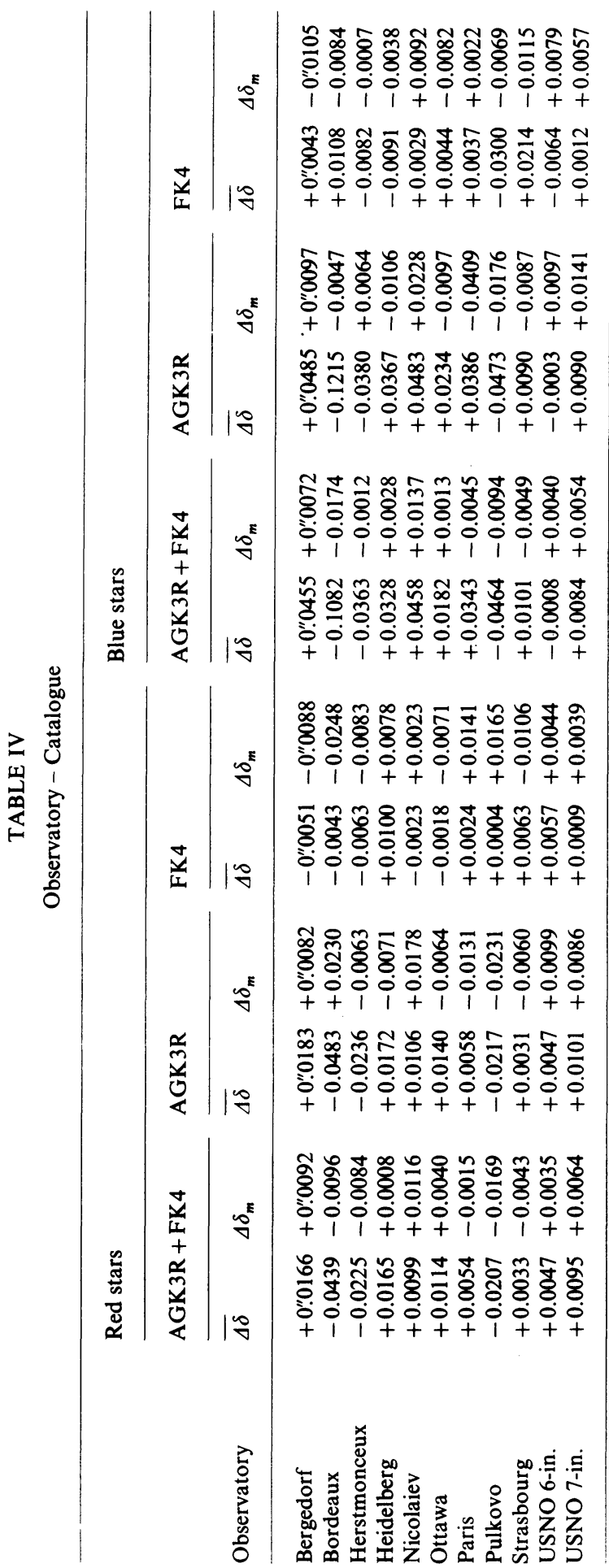


FK4 stars, using FK4 proper motions. The results are shown in Table V and Figures 7 and 8. In right ascension, although small, the rate is 3.5 times its mean error, while in declination the rate is insignificant.

\section{TABLE V}

FK4 and proper motion system-catalogue

\begin{tabular}{lllllr}
\hline & $\overline{\Delta \alpha}$ & $\Delta \alpha_{m}$ & $\overline{\Delta \delta}$ & $\Delta \delta_{m}$ & \multicolumn{1}{c}{$N$} \\
\hline FK4 - Cat. & +0.0005 & -0.0052 & +0.0003 & +0.0009 & 886 \\
PMS - Cat(FK4) & -0.0039 & -0.0026 & -0.0025 & +0.0005 & 870 \\
PMS - Cat(AGK3R) & +0.0016 & +0.0052 & +0.0061 & +0.0040 & 5394 \\
PMS - Cat(AGK3R + FK4) & +0.0009 & +0.0024 & +0.0049 & +0.0030 & 6264 \\
By average magnitude groups & & & & & \\
PMS - Cat(AGK3R +FK4) & +0.0062 & -0.0009 & -0.0023 & +0.0008 & 6264 \\
\hline
\end{tabular}

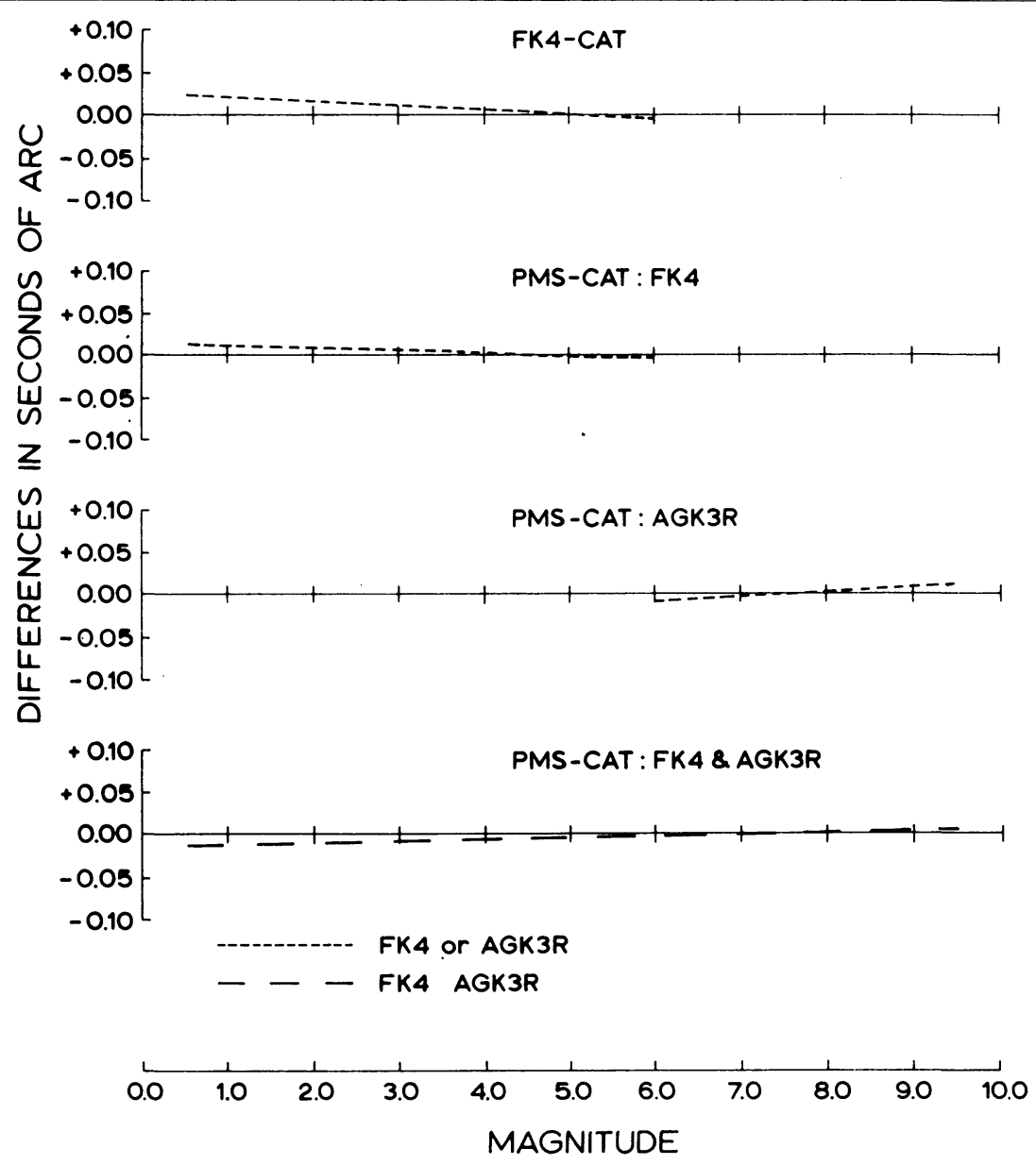

Fig. 7. Right ascension. 


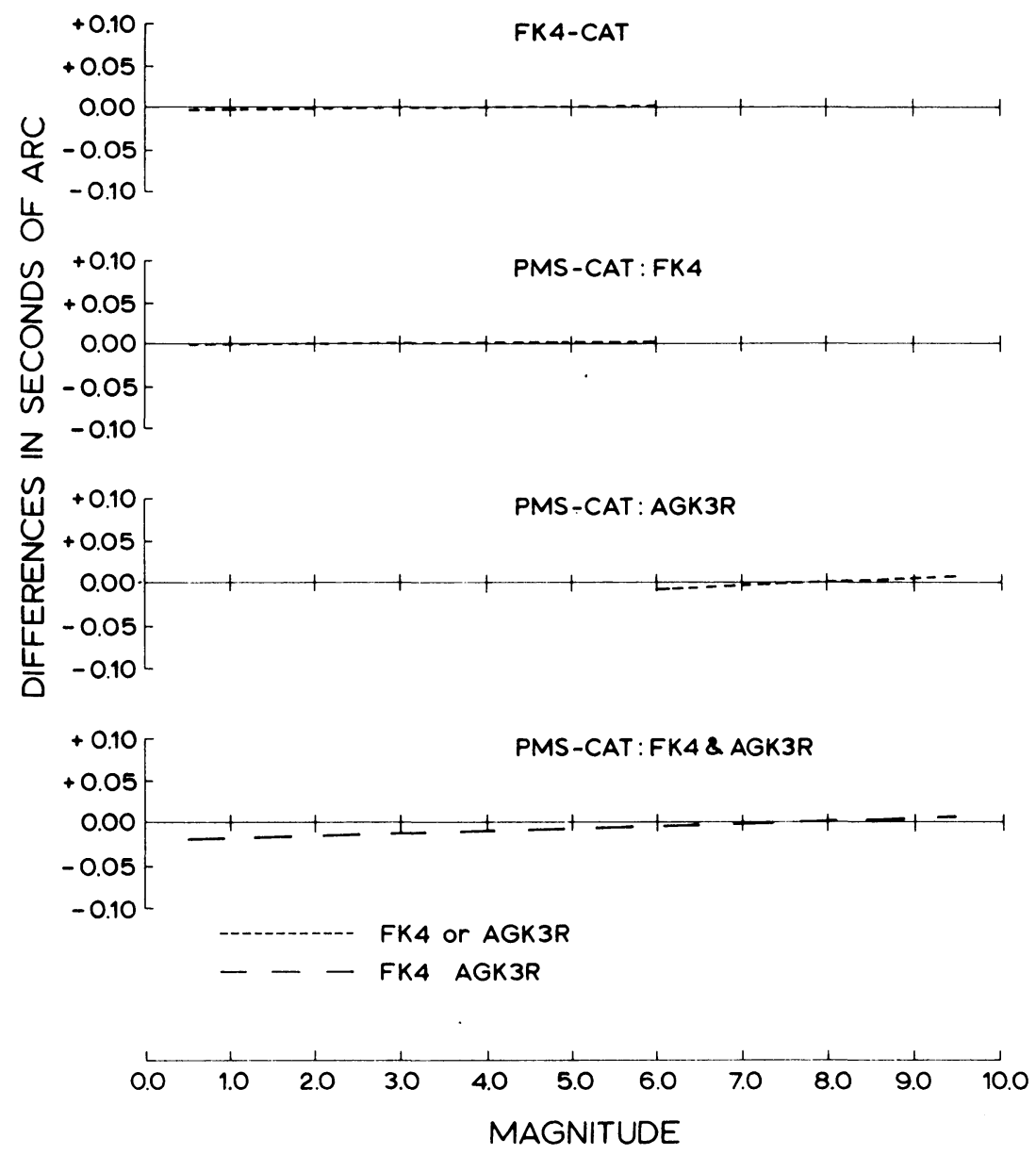

Fig. 8. Declination.

\section{Comparison with the AGK3R Proper Motion System}

In deriving proper motions for the AGK3R stars, a basic proper motion system(PMS) is being developed that will be used to bring in more and more of the older catalogues. Carefully selected catalogues that are relatively free of magnitude effect and that had been observed in such a way as to avoid other problems now known to exist in 19th century catalogues were used to form this base system of some 5400 reference stars. In order to compare the AGK3R Catalogue with some other standard, differences were formed between the PMS and the AGK3R at the epoch of the AGK3R observations. This was done for 3 groups: FK 4 stars, AGK3R stars and both of these groups combined. While it is realized that the PMS includes the AGK3R, it also includes nine other catalogues ranging in epoch from 1898 to 1958. The mean epoch of the PMS is about 1935. The results of this comparison are shown in Table V and 
Figures 7 and 8 . In declination, the rate generated by the comparison of the observed FK4 stars with the PMS is near zero and, in right ascension, it is about half that of the comparison with the FK4 itself. Whether this is due to a magnitude effect in the FK4 is uncertain, since, as noted above, the AGK3R is one of the constituents of the PMS.

\section{Comparison by Average Magnitude Groups}

All of the above analyses have been made by star, that is, each star received equal weight, regardless of its magnitude. This means that in the analyses of the combined FK4 and AGK3R stars, the fainter magnitudes entered into the solution with considerably greater weight than the brighter magnitudes, due to the distribution of stars. An analysis was also made, collecting the differences for each half magnitude for (PMS - AGK3R + FK4). A solution was made giving each of these groups equal weight. The results are shown in Table $\mathrm{V}$. There is little evidence of a significant magnitude effect.

\section{Conclusion}

There is little evidence of a significant magnitude effect in the AGK3R. If any, it is less than $0.01 \mathrm{mag}^{-1}$ in either coordinate.

\section{DISCUSSION}

Van Herk: What magnitudes have been used? The nominal values or the apparent magnitudes as they were during the observations? If the first, you are bound to get too small values for the 'magnitude' effect, perhaps up to $100 \%$.

Klock: I don't know which values were used. The authors of the paper would have to be consulted before answering the question.

Eichhorn: In my judgement, it would be necessary to include in the meridian catalogues and photographic catalogues more early type (or blue) stars than there are now. There is no doubt that the existing star catalogues are afflicted by systematic errors which depend on the color index of the stars in them. These errors can be reliably determined only if there are enough early type stars in the catalogue; otherwise these errors may be difficult to separate from random errors.

Since it is of utmost importance, in questions of galactic kinematics, that there are no color dependent systematic errors in the proper-motion material, I believe it would be advantageous if the compilers and observers of star catalogues would make a conscious effort to include more blue and white stars than there are now, so that it will become possible to determine reliably the color-dependent systematic errors also for the stars at the blue end of the spectrum.

Klock: Schombert and Corbin investigated the possibility of a magnitude or color effect and their results are given in Table III and IV of their paper. Their conclusion was that there was no significant relationship with color.

In regard to the inclusion of more early type stars in meridian circle programs it should be mentioned that the last two Washington 6-in. observing programs included the Blaauw stars, giving about $15-20 \%$ early type stars in their list. 